\title{
Las chicas del cable en huelga. Feminismo peruano Zoila Aurora Cáceres y el reclamo de las telefonistas (Lima, 1931)
}

\author{
The cable girls on strike. Peruvian Feminism Zoila Aurora \\ CÁceres and the operators' Claim (Lima, I93 I) \\ Sofia Pachas Maceda \\ Universidad Nacional Mayor de San Marcos, Lima, Perú \\ http://orcid.org/0000-0001-8736-8534 \\ spachasm_ac@unmsm.edu.pe
}

Resumen: En noviembre de 1930, la escritora peruana Zoila Aurora Cáceres volvió a Lima desde Europa. Este regreso marcó la continuación de la campaña que había inaugurado años antes, cuando fundó el colectivo Feminismo Peruano en 1924, y que retomó con más fuerza en el marco de la Asamblea Constituyente de 1931. En ese contexto, un grupo de telefonistas encontraron en Cáceres una valiosa aliada en su reclamo por un incremento salarial y un mejor trato laboral. Esta alianza confirma lo que Michelle Perrot señala al referir que las primeras iniciativas de sindicalización femenina fueron organizadas por mujeres ajenas al mundo obrero, pero comprometidas con el movimiento asociativo y de reivindicación. El artículo aborda la huelga desde la perspectiva de género en la historia, para lo cual se utiliza el término "agente histórica" con el objetivo de destacar la capacidad de acción y las tácticas de las que hicieron uso tanto las telefonistas como Cáceres.

Palabras clave: Zoila Aurora Cáceres, sindicalismo, huelga, telefonistas, Perú 1931. 
AвSTRACT: In November of 1930 the Peruvian writer Zoila Aurora Cáceres went back to Lima from Europe. This return left a mark in the continuity of the campaign opened years before, when she founded the Collective Peruvian Feminism in 1924 and which resumed with more strength in the context of the Constitutional Assembly of 1931. Against this background, a group of telephone operators found in Caceres a valuable allied for their salary raise and a better working environment complaint. This alliance confirms what Michelle Perrot points it out when referring to the first initiatives of female unionization, organized by women external to laborer world but compromised with the associative movement and recognition. The article presents the strike from the gender approach in the history. For which the word historical agent is used to highlight the capacity of action and the tactics that use both the telephone operators and Caceres.

KeYwords: Zoila Aurora Cáceres, syndicalism, claim, operators, Perú 1931.

"Fuera represión" ${ }^{1}$ CÁCeres y las peruanas en movimientos SOCIALES ${ }^{2}$

Desde fines del siglo XIX, Zoila Aurora Cáceres Moreno (Lima, 1872-Madrid, 1958) (Figura 1) empezó a escribir sobre la condición social de la mujer, lo que se materializó en su tesis El feminismo en Berlín (circa 1906) y en su primer libro, Mujeres de ayer y hoy (1909). Esto confirma que, tanto desde el relato histórico como desde la ficción, la mujer se convirtió en el eje de su escritura y en su labor de activista ${ }^{3}$.

1 Las chicas del cable es el título de la primera serie original de Netflix producida en España (2017-2020). Este artículo ha sido, en parte, inspirado en el capítulo 12, del que toma algunas palabras de los diálogos para dar nombre a dos epígrafes.

2 Esta investigación fue financiada por la Universidad Nacional Mayor de San Marcos - RR N ${ }^{\circ}$ 005753-2021-R/UNMSM con código de proyecto E21031021.

3 Hija de Andrés Avelino Cáceres y Antonia Moreno, dos importantes referentes de la historia peruana republicana, Zoila Aurora fue una importante intelectual de entre siglos. Publicó seis libros e innumerables artículos dados a conocer en periódicos y revistas peruanas y extranjeras. En 1906, fundó en Lima el Centro Social de Señoras. cuyo objetivo era brindar a mujeres de escasos recursos económicos la posibilidad de aprender un oficio. Esta fue su primera iniciativa 


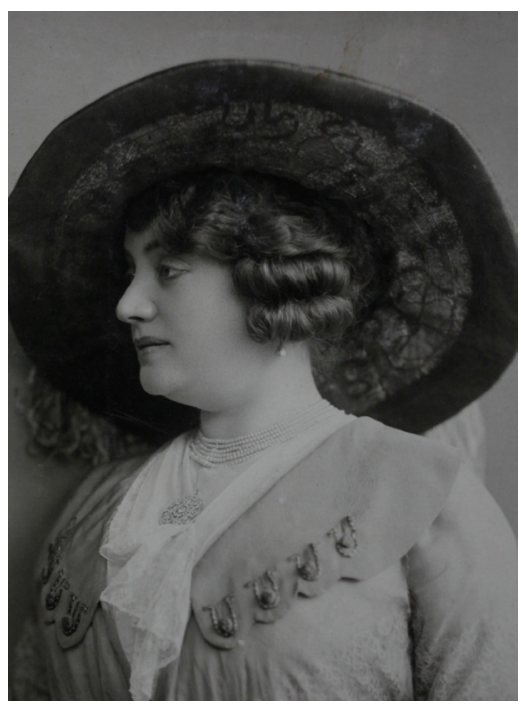

Figura i. Zoila Aurora Cáceres. Álbum localizado en el Museo Andrés Avelino Cáceres.

En Perú, como en otros países del mundo, el siglo XX inició sin mayores cambios en lo referido a la ciudadanía de las mujeres. No obstante, cada vez más se dejaban escuchar voces de cambio a favor de ellas, lo que implicó la discusión sobre sus derechos como ciudadanas, pues deberes hacía mucho tiempo que tenían. La primera propuesta seria desde el feminismo la elaboró María Jesús Alvarado, cuando fundó en Lima el movimiento Evolución Femenina (1914). Alvarado planteaba una necesaria "toma de conciencia" de las peruanas para que lograran progresar en campos como el educativo, el laboral y su consecuente independencia económica, además del fundamental "derecho a ser electora y elegible" (Zegarra 239).

Todavía faltaba mucho camino para mejorar en los puntos señalados, pero en el ámbito laboral de las dos primeras décadas del siglo XX ya habían empezado a realizarse esfuerzos por hacer escuchar sus reclamos.

para revertir la situación social de las peruanas, la misma que se convierte años después en su colectivo Feminismo Peruano. En los últimos años, sus facetas de literata, viajera y activista política están siendo de interés de investigadores que están revalorando su aporte a las letras y a la lucha feminista peruana. 
En 1901, se celebró en Lima el primer Congreso Obrero. Tres años después, un sector anarquista se separó de la Confederación de Artesanos y formó la Federación de Obreros Panaderos "Estrella del Perú" y con ello se da inicio al movimiento sindical propiamente dicho (Basadre 124).

En ese ambiente de reclamos laborales, una de las más emblemáticas luchas fue la emprendida por los jornaleros de Huaura y Sayán -costa central de Lima- en 1917, movilización que tuvo como escenario un mundo que vivía las consecuencias de la Primera Guerra Mundial, lo que había recrudecido las condiciones de vida de los trabajadores -que ya eran duras antes del conflicto bélico-. Al respecto, Josefina del Prado señala que, si bien en el transcurso de la guerra el Perú se favoreció por las exportaciones, al finalizarla los problemas de "la carestía de vida" se agudizaron debido a la aparición de monopolios y de una corriente de importaciones que debilitaron el desarrollo de la industria nacional (213-4).

Esto desencadenó, en junio de 1917, una paralización total de dieciocho días que comprometió a los jornaleros y a la cadena de distribución de productos del valle. Un grupo de mujeres se sumó a esta movilización, pues muchas de ellas eran compañeras de los huelguistas pero también vendedoras en los mercados de la zona, por lo cual hicieron suya la protesta. A diferencia de la huelga de los trabajadores textiles de Vitarte de 1914, en la que las mujeres habían sido principalmente voceras y encargadas de recaudar víveres y dinero para el sostenimiento del paro (Both 97), la intervención de ellas en el conflicto de Huaura y Sayán fue más comprometida y arriesgada, pues tuvo un saldo de, por lo menos, dos mujeres muertas tras el enfrentamiento con la tropa, lo cual "representó la primera acción femenina organizada en apoyo a las luchas sindicales" ${ }^{4}$ (Carlessi 124).

Mientras esto sucedía en las afueras de Lima, en la capital se producía desde inicios del siglo XX lo que Maritza Villavicencio identifica como "la vertiente urbano popular" (36). Vinculada al crecimiento industrial, la mano de obra femenina encontró posibilidades de laborar como obreras y empleadas en oficios relacionados a telégrafos, correos y telefonía.

4 Por lo significativo del hecho, se cree importante colocar el nombre de estas primeras víctimas de la lucha por los derechos de los trabajadores: Irene Salvador de Lino y Eulogia Morales. 
Es probable que algunas de esas trabajadoras apoyaran el movimiento que en enero de 1919 llevó a la obtención de la jornada de ocho horas para todos los trabajadores, y que, meses después, derivó en una huelga general contra el alza del costo de vida. Una vez organizado un Comité Pro-abaratamiento de las subsistencias, se procedió a la elección de un comité femenino que llevó a cabo "la primera asamblea de mujeres de la historia social peruana" (Villavicencio 39) y en donde el nombre de Zoila Aurora Cáceres empezó a vincularse con las luchas de las trabajadoras, dado que presentó la propuesta para la organización de una marcha de mujeres. Empezaban a formarse espacios de encuentro entre intelectuales comprometidas con la reivindicación de las peruanas, como fue el caso de la abogada Miguelina Acosta Cárdenas (Rojas 13), quien tiempo después colaboraría con Feminismo Peruano.

Zoila Aurora Cáceres pasó de la propuesta a la acción cuando en 1924 fundó Feminismo Peruano. Gracias a sus vínculos sociales e intelectuales, este colectivo tuvo una amplia cobertura en los medios de prensa que publicaron en extenso los objetivos del grupo, que buscó revertir los abusos del todavía vigente Código Civil de 1852.

En respuesta a las limitaciones de la libertad de las mujeres, Feminismo Peruano propuso tres reformas: en la ley -el derecho a la ciudadanía de todas las mujeres-, en el Código Civil -poder ser guardadoras, testigos testamentarios, fiadoras y mandatarias judiciales- y en el matrimonio - ejercer la patria potestad y administrar su patrimonio, contraer nupcias a partir de los dieciocho años, libertad de contratación y la investigación de la paternidad- (Cáceres, "Programa" 9).

Esta fue la primera etapa de Feminismo Peruano, que puede considerarse como de sensibilización y difusión de ideas dirigidas a cambiar el pensamiento de los dirigentes y, en especial, el de las peruanas. Pero, en 1931, una campaña sin precedentes fue impulsada por el grupo rebautizado como "Feminismo Peruano Zoila Aurora Cáceres"5 (FP-

5 Sobre el cambio de denominación, al adicionar su nombre a la del colectivo, la misma Cáceres lo explica en una carta publicada en el periódico El Pueblo. Allí comentó que, ante su ausencia del país, unas socias de Feminismo Peruano querían cambiar la orientación y objetivos del grupo, ante lo cual ella se sentía en el derecho de fundar otro Feminismo Peruano y por ello añadió su nombre, para diferenciarlo del que fundó en 1924 (Cáceres, "Feminismo Peruano. Contestación” 11). 
ZAC). Esta decisiva etapa marcó una alianza con civiles, pero también con grupos de diversa índole, entre los cuales el de los trabajadores(as) fue el más relevante.

Habitual viajera que solía radicarse en Europa, Cáceres llegó a Lima en noviembre de 1930. Su arribo fue planificado a partir del tenso contexto que vivía el Perú luego del derrocamiento del presidente Augusto B. Leguía y de la posibilidad de introducir cambios relevantes en la nueva Constitución que se anunciaba.

Durante sus once años en el poder (1919-1930), la estrategia fue cambiando según los intereses del gobernante. Al principio, Leguía buscó concertar con las mayorías -obreros e indígenas- que habían sido relegadas por los gobernantes del Partido Civil. No obstante, transcurridos los primeros años y asegurados sus allegados en puestos claves dentro del Poder Ejecutivo y Legislativo, esos vínculos entre Leguía y los olvidados fueron dejados de lado.

Algo que contribuyó a reforzar la idea de una propuesta política renovadora fue el uso del lema "Patria Nueva", que en materia económica implicó un proceso de modernización e industrialización del país a través de la presencia del capital extranjero, en especial, estadounidense. Con este estrecho vínculo y con una dinámica de empréstitos que llevaron a elevar la "deuda externa de 25 millones de soles en 1919, hasta los 221 millones diez años después" (Contreras y Cueto 247) es que Leguía llega a proclamarse, por tercera vez consecutiva, como presidente.

Su régimen autoritario no dudó en perseguir y reprimir a sus adversarios, entre los cuales también se encuentra la ya mencionada María Jesús Alvarado. Ella tuvo que dejar el país luego de negarse a brindar los nombres de los obreros opositores al régimen que habían elaborado volantes en su imprenta (Zegarra 25). El desgaste del mandato de Leguía fue creciendo a medida que transcurría la década de 1920, y su desenlace se aceleró con el colapso de la Bolsa de Valores de Wall Street, el 24 de octubre de 1929, y con ello la reducción de las exportaciones peruanas, la rebaja de sueldos y el desempleo.

Luego de diez meses de iniciada esta crisis internacional, el descontento y los reclamos de diversos sectores de la sociedad se hicieron insostenibles, lo que desencadenó la revolución del 22 de agosto de 1930, encabezada por el comandante Luis Sánchez Cerro, cuya sublevación 
ocurrió en Arequipa, al sur del Perú. Desde allí, instaló una Junta Militar de Gobierno que duró seis meses, en los que la tensión continuó en diversas ciudades, ante lo cual Sánchez Cerro decidió alejarse del poder y dar paso a una Junta Nacional de Gobierno, que sería la que llevaría a cabo las elecciones para la Asamblea Constituyente y la presidencial (Padilla 100).

El político cusqueño David Samanez Ocampo ejerció la presidencia de manera interina durante nueve meses hasta que, luego de ganar las elecciones democráticas, Sánchez Cerro asumió el mandato. Además de adoptar algunas medidas para sobrellevar el desempleo y el ingreso de escasos recursos, tales como el otorgar bonos de alimentos a obreras y costureras (Padilla 103), la principal misión de esta Junta Nacional estuvo enfocada en llevar a cabo el proceso electoral.

En esta coyuntura entre la Junta Militar de Sánchez Cerro y la Junta de Gobierno encabezada por Samanez, Zoila Aurora Cáceres vuelve a poner en debate el voto de la mujer en el Perú. Sin embargo, esta vez contaría con una red de adhesiones que la llevó a descentralizar la lucha a partir de una campańa llevada a cabo entre noviembre de 1930 y enero de 1932. Para ello, habilitó una red de conexiones donde una activa correspondencia entre Lima y los departamentos de Ancash, Cerro de Pasco, Cusco, Junín, Huancavelica, Ica, La Libertad y Lima ${ }^{6}$. Gracias a estas misivas es posible saber el alcance nacional del movimiento generado por Cáceres, pero también el nombre, la palabra e ideas de un numeroso grupo de mujeres y hombres que se sumó a los propósitos de FPZAC (Pachas 51).

La primera meta del colectivo fue insertar el asunto del sufragio femenino en el Estatuto Electoral, pero, al no conseguirlo, la lucha se centró en lograr que este propósito fuese aprobado en la Asamblea Constituyente. Para ello, apoyó la candidatura de aquellos que manifestaban, públicamente y por escrito, su postura a favor del voto de las peruanas.

Durante los últimos y ajetreados meses previos a la elección de los representantes de la Asamblea, las energías de Zoila Aurora Cáceres se dividieron, al coadyuvar a otra lucha que por esos meses empezó a librarse entre un grupo de trabajadoras y una empresa transnacional.

6 En la década de 1950, Cáceres donó esta correspondencia a la Biblioteca Nacional del Perú. 
La primera comunicación entre las trabajadoras y la líder del colectivo se remonta al 30 de junio de 1931, cuando 59 telefonistas solicitan a Cáceres que interceda por ellas, anunciando la visita de una comisión a fin de detallarle los motivos de su reclamo. Las telefonistas confiaban en que Cáceres las apoyaría, en tanto ellas se comprometían a acatar "los arreglos y resoluciones que Ud. tenga a bien adoptar, seguras como estamos, que ellas nos serán favorables" (Martínez de la Torre 210). Luego de estas líneas en las que una relación jerárquica se impone al utilizar el verbo acatar, solicitan se las considere como socias de FPZAC. De esta manera, se busca equilibrar la situación, pues se sellaba una alianza simbólica en la que todas serían beneficiadas: las telefonistas, al lograr la representatividad de una figura pública como Cáceres, y a su vez el colectivo, al adherir más voces asociadas a la lucha de las mujeres.

Este fue el inicio de una sinergia de voluntades que se unieron en pro de un reclamo justo que mantuvo a Lima, entre el 26 de agosto y el 28 septiembre de 1931, atenta a las noticias concitadas por las telefonistas en huelga.

“Las telefonistas no están solas". El decisivo apoyo de Feminismo Peruano ZaC.

En Perú, fue durante el primer gobierno de Andrés Avelino Cáceres ${ }^{7}$, padre de Zoila Aurora, que se consideró como asunto de interés general lo relativo al desarrollo del servicio telefónico. Para ello, se expidió el decreto del 16 de noviembre de 1886, mediante el cual se anunció la licitación pública de los servicios telegráficos y telefónicos. Sin embargo, ante el fracaso de esta convocatoria, dos años después se declaró el libre establecimiento de las líneas (Huamani 15).

En 1930, transcurridos algunos años en los que el capital nacional había sostenido la empresa telefónica, los socios de la Compañía Peruana de Teléfonos vendieron la mayoría de las acciones a la International Telephone \& Telegraph Corporation, con el objetivo de modernizar el

7 Luego de la resistencia que lideró durante la Guerra del Pacífico (1879-1883), Andrés A. Cáceres fue presidente del Perú en dos oportunidades, de 1886 a 1890 y de 1894 a 1895. 
servicio (Huamani 36). Un año después, esta transnacional estadounidense, que contaba con 25 filiales en diversas ciudades del mundo, mantenía conectados a abonados de 31 ciudades del norte, centro y sur del Perú. Para 1930, es decir, un año antes de la huelga, los usuarios superaban los nueve mil ("El servicio telefónico" 10).

Antes de exponer las demandas de las telefonistas, es necesario señalar el escaso interés que ha recibido esta huelga en la historiografía peruana, pues en la mayoría de los casos se trata de breves menciones a propósito de la colaboración de Zoila Aurora Cáceres (Basadre 76; Bolt 150; Villavicencio 35). El primero en otorgarle el interés que merece esta protesta fue el escritor y político de filiación socialista, Ricardo Martínez de la Torre, quien le dedicó varias páginas del cuarto tomo de su libro Apuntes para una interpretación marxista de la historia social del Perú (1949). Aunque el autor no elabora un texto sino que transcribe los volantes, comunicaciones y artículos de periódicos publicados a raíz de esta huelga, su aporte es significativo, ya que a través de este trabajo compilatorio dejó constancia de la lucha de las telefonistas.

Una investigación más reciente de Cristian Padilla la considera entre las manifestaciones de descontento social surgidas durante la Junta de Gobierno de Samanez y le dedica una página de su tesis a este conflicto en el que, más que referirse a las huelguistas, se detiene en la intervención que los representantes del gobierno central y local tuvieron en este conflicto (107).

A este escaso interés de investigaciones anteriores se debe el objetivo de este artículo de dar a conocer esta huelga de forma más detallada, donde las trabajadoras fueron las protagonistas y no parte del grupo de apoyo de una protesta organizada por hombres. Este planteamiento se inscribe en los estudios de género en las ciencias sociales, en el sentido de dar a conocer procesos históricos en los que se ponen en evidencia la subordinación social y política de las mujeres, pero, en especial, las tácticas que utilizaron para hacer valer sus propuestas. Entiéndase "táctica" en el sentido otorgado por el historiador Michel de Certeau que utiliza este vocablo para referirse al "arte del débil", de los sometidos, quienes emplean determinadas "acciones delimitadas" por el campo de visión del enemigo (Pellegrini, "Huelgas" 310$)^{8}$.

8 La investigadora Micaela Pellegrini hace uso de este concepto en su interesante análisis de la huelga de maestras de la localidad de Firmat (Argentina, 1921), para 
Asimismo, para llevar a cabo el propósito de visibilizar la lucha femenina, se hace uso aquí del término "agente" en alusión a la capacidad de acción de un agente social inserto en una trama de posibilidades en las que puede continuar la pauta trazada o reaccionar de una manera distinta. A decir de Rosa Belvedresi, a esta determinación se le une que las mujeres, como "agentes históricas", están determinadas -limitadaspor los recursos a los que puedan acceder en distintos contextos. Ante ello, la autora hace hincapié en los casos en que las mujeres se alejan de los "moldes pre-establecidos" y con ello generan líneas de acción que no son esperables para ellas (8). Al respecto, no es casual que Belvedresi mencione como ejemplos de esas inesperadas incursiones femeninas a las luchas por el sufragio y las jornadas laborales.

Un mes antes de la huelga de las telefonistas fue publicado el artículo "La movilización política de las mujeres", del poeta indigenista y periodista cajamarquino, Nasario Chávez y Aliaga. En dicho texto, su autor reflexionó sobre la capacidad de las mujeres "prontas a convivir las luchas del proletariado y a ofrecer a él sus reservas de energías pero abominables exclusivismos, vetustos privilegios [...] han impedido que la mujer participe, de modo alguno en la contienda social" (11)

Interesante postura que critica las ideas prejuiciosas dentro de las organizaciones de trabajadores y que bien podría tener origen en un reclamo social que acababa de suceder en otro lugar del mundo y cuyas noticias, sin duda, llegaron a oídos de un hombre ilustrado como Chávez. Se trata de la huelga de telefonistas en Madrid, iniciada el 4 de julio de 1931 y que es considerada como uno de los primeros conflictos de carácter social importante que tuvo que hacer frente la Segunda República Española (De la Cruz 2015).

Por su parte, en Latinoamérica, previo al reclamo de las telefonistas limeńas, se tiene noticias de dos huelgas: la llevada a cabo en Uruguay en 1922 y la de Bogotá en 1928. Esta última, de seis días, contó con un apoyo decisivo de la ciudadanía, que contribuyó aportando dinero, difundiendo carteles e, incluso, al "bloquear la entrada de las instalaciones de la Compañía" (Felacio 45). En tanto, con sus respectivas particularidades, la huelga de telefonistas uruguayas tuvo mayor cercanía con la de Lima, pues fue una líder feminista, Paulina Luisi, quien fundó la del magisterio para invalidar opiniones adversas a sus reclamos ("Huelgas" 310). 
Unión Nacional de Telefonistas. Esta huelga contó con el apoyo de otros sindicatos y duró más de un mes (Yael y Darré 283).

La primera noticia sobre la huelga de telefonistas en Lima fue dada a conocer el 26 de agosto. Ese día, El Comercio informó que las trabajadoras habían entregado un pliego de reclamos a la gerencia. Además, se publicaron declaraciones y una carta del gerente Fernando Carbajal, quien señaló que la huelga había sido organizada al conocerse que un grupo de telefonistas serían separadas de su cargo debido a la apertura de una planta automática de teléfonos ("La huelga de telefonistas" 1). Sin embargo, conforme pasaron los días, la opinión pública conocería que el despido de estas trabajadoras fue el último episodio de una serie de maltratos que la compañía llevaba a cabo de manera sistemática contra las operarias.

Algo que sobresale en esta primera noticia fue que, en la misma página y al lado del artículo, se dispuso un aviso de la Compañía Peruana de Teléfonos, en el que se convocaba a que antiguas operadoras y practicantes se presentasen a la empresa. De esta manera, la compañía dejó notar sus pocas intenciones de negociar, pues lo que pretendía era ignorar el reclamo y continuar con el servicio, valiéndose del trabajo de otras operarias. Ese paralelo visual no deja lugar a dudas sobre la desigualdad de fuerzas que iban a medirse esos días de huelga. Así, mientras un breve y nada atrayente artículo daba a conocer la noticia del paro de las telefonistas, un aviso, de mayor formato y letras grandes, ofrecía empleo a antiguas operarias y practicantes (Figura 2).

Otro detalle que se aprecia en la lectura de esta primera noticia es que no se identificó a una líder, nadie que las represente dentro de las filas de las trabajadoras. El único nombre que destacó en todos los días de la huelga fue el del gerente Fernando Carbajal. 


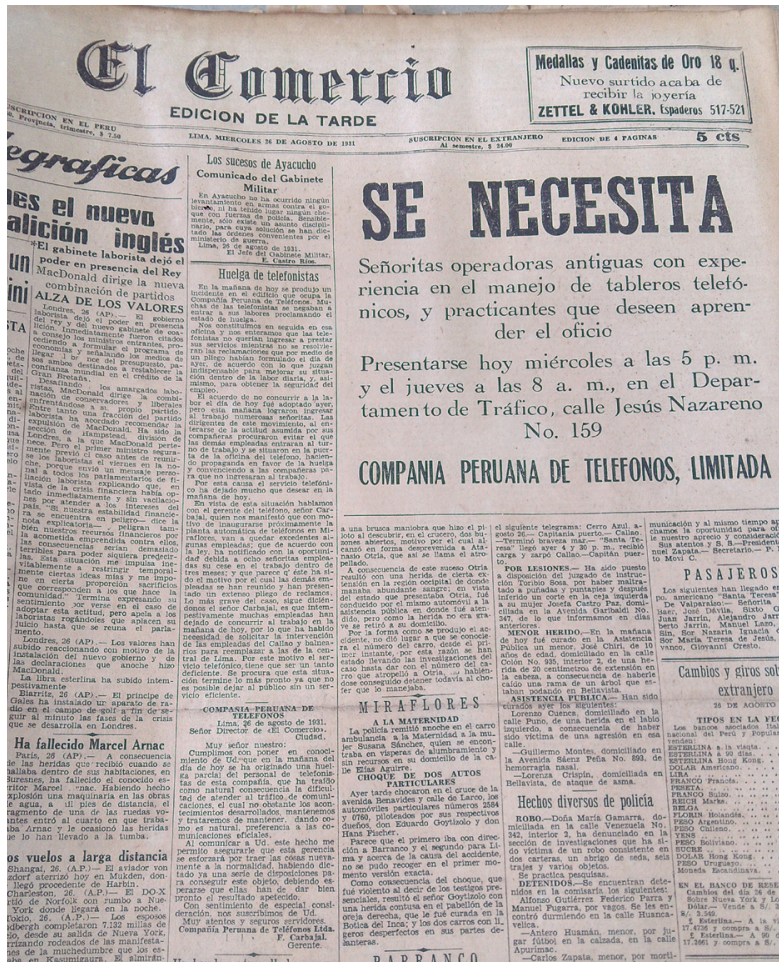

Figura 2. Portada del periódico El Comercio, 26 de agosto de 1931.

La contraparte de esta voz masculina fue Zoila Aurora Cáceres, quien asumió la defensa de 104 telefonistas ("Continua sin solución la huelga” 11). Su intervención fue fundamental, pues en esos meses de campaña por el voto de la mujer su nombre captaba la atención de los medios, siendo una líder de opinión cuya postura era siempre de interés?. Lejos de ser un apoyo circunstancial, fue Cáceres quien asesoró a las

9 El interés por tener el apoyo de Cáceres se reflejó también en una misiva que le envió Eudocio Ravines, secretario del Partido Comunista Peruano, donde le solicitó que se pronunciara a favor de una amnistía para los marinos chilenos que se habían sublevado en Coquimbo y que, luego de ser apresados, corrían peligro de muerte (Ravines 1). Cáceres accedió a la solicitud y remitió una carta solidarizándose con este caso, de ello ha quedado registro el documento de recepción de la Embajada de Chile en Perú. 
huelguistas e incluso redactó un documento que fue enviado a la Junta de Gobierno el 20 de agosto, es decir, una semana antes que se hiciera pública la protesta ${ }^{10}$. El referido documento inicia así:

En mi condición de presidenta del "Feminismo Peruano ZAC" institución que defiende los derechos de la mujer, y en virtud de la Ley $2851^{11}$, art. 31 , que autoriza a las instituciones a vigilar la ejecución de las leyes obreras, pido a ese Ministerio que se ampare a las señoritas telefonistas contra los abusos que la Compañía Peruana de Teléfonos Ltda., comete con sus empleadas, no obstante de que la Ley $\mathrm{N}^{\circ} 2851$ sobre trabajo de mujeres y nińos, por cuenta agena [sic], es bastante limitada, por lo que no demanda gran esfuerzo el cumplirla ("La huelga de telefonistas" 12).

Su defensa la somete, punto por punto, a lo contemplado por la ley, pero también a algunas modificaciones posteriores, lo que le otorga una solvencia indiscutible. De igual manera, días después, redactó el pliego de reclamos con catorce puntos que serían los tomados en cuenta para negociar el fin de la huelga.

No era casual que Cáceres tuviera conocimiento de los derechos laborales, dado que FPZAC mantenía correspondencia con la Alianza Internacional de Mujeres por el Derecho de Sufragio e Igual Ciudadanía, ente internacional no gubernamental creado en 1904 por la feminista estadounidense Carrie Chapman Catt. La organización solicitaba a sus adherentes datos sobre varios aspectos de la vida de las mujeres y, precisamente, uno de los cuestionarios localizados en la correspondencia de Cáceres se refiere a las condiciones laborales de las trabajadoras. Por ello, es probable que estuviera al tanto de dichas leyes cuando las telefonistas solicitaron su apoyo.

En este sentido, un dato relevante es que las telefonistas no estaban sindicalizadas cuando iniciaron su reclamo. Esta situación fue utilizada

10 En una carta enviada a El Comercio, Cáceres señaló que dos meses antes del inicio de la huelga, ella había conversado con los directivos "americanos" en su búsqueda de conciliar antes de llegar a medidas más drásticas (Cáceres, "Sobre la huelga" 12). Esto demuestra el nivel de compromiso que tuvo en este conflicto.

11 Ley promulgada el 23 de noviembre de 1918, durante el gobierno de José Pardo. 
por los directivos de la Compañía de Teléfonos para negarse a negociar y por ello la huelga se dilató. Este punto es interesante de analizar dado que Cáceres, lejos de convencerlas de desistir, las alentó en su reclamo y con ello dejó de lado uno de los puntos centrales en la resolución de conflictos laborales: el contar con un sindicato identificado por el empleador. No obstante, esta aparente desventaja de las huelguistas hizo que diferentes gremios y muchos miembros de la comunidad, incluso abonados, se solidarizaran con las telefonistas y con ello se aprovechó esa posición subalterna. Apoyo que debe haberlas alentado, pues "hacer huelga implica desafiar la opinión y comportarse como mujeres públicas" (Perrot 164).

Iniciada la huelga, se procedió a la redacción de volantes, un recurso que posiblemente haya sido sugerido por la líder de FPZAC. Del primer volante que circuló se extraen los siguientes párrafos:

Ante esta grave situación las empleadas de teléfonos, no hemos tenido más recurso que declararnos en huelga en defensa de nuestros derechos pisoteados día a día por elementos extranjeros en confabulación con un peruano designado como nuestro jefe [...] Esperamos que el público de Lima, la opinión sensata y en fin, todos aquellos que saben lo que es el dolor de trabajar por un mísero salario, sabrán responder a nuestro llamado, prestándonos todo su apoyo ("Continua sin solución la huelga" 11).

El primer párrafo pone en consideración un punto que tomará relevancia en la huelga: el abuso de una empresa extranjera. Ante este trato irregular a los connacionales, algunos opinaban que, al ser la telefonía un servicio público, las ganancias estaban aseguradas y por ello era un negocio lucrativo que debía ser nacionalizado. Esta idea fue difundida por Ernesto Vidaurre, uno de los más leales seguidores de esta huelga. Vidaurre apoyó la movilización redactando artículos en los que da a conocer reveladoras cifras que resaltan las diferencias salariales entre una telefonista que cobraba $4 \mathrm{Lp}^{12}$ y el controlador que recibía $150 \mathrm{Lp}$ (Vidaurre 6). Abismales distancias salariales que ponen en relieve los diferentes honorarios de acuerdo al sexo, al considerarse que los hombres debían recibir un sueldo mayor debido a que eran los jefes de familia.

12 Este monto en libras peruanas equivalía a 45 soles. 
En lo referido al llamado a que se solidaricen con su lucha, es posible apreciar la impronta de Zoila Aurora en los volantes de Feminismo Peruano, pues solía hacer lo propio. En uno de ellos escribió:

Ejército y Armada hoy no tienen enemigos. Vosotros que sabéis ser valerosos en los campos de batalla. Calmad vuestro ardoroso celo. El combate del momento es otro, necesitáis triunfar dentro de la virtud cívica que reclama imperiosamente paz y amor.

Mujeres todas, acompañad al "Feminismo Peruano ZAC" en su vehemente deseo (Cáceres, "Manifiesto" 2).

Con una retórica nacionalista, la lideresa de FPZAC aludía al recuerdo de la Guerra del Pacífico (1879-1883), a la que su apellido estaría siempre asociado por la resistencia encabezada por su padre en la conocida Campaña de la Breńa. Esa remembranza, en el contexto de la lucha por el voto de la mujer, tomó otro cariz. La batalla era, entonces, contra las ideas y prejuicios que querían continuar con "la esclavitud"13 de las peruanas. En tanto, para las telefonistas se coincidía en la convocatoria de unión contra un enemigo, que al igual que en la guerra, se trataba de un poder extranjero.

Pero las huelguistas también agenciaron otros respaldos que contribuyeron en la negociación, como el Sindicato de Empleados Bancarios, que asumió la defensa a los pocos días de iniciada la protesta ("Continua sin solución la huelga” 11). Si bien la experiencia sindical era necesaria, también es posible vincular este apoyo a una táctica para obtener mayores beneficios, dado que tanto los representantes del Estado como los directivos de la Compañía de Teléfonos eran hombres. Asunto que pone en relieve la falta de representatividad de las mujeres dentro de los sindicatos, pero también su principal obstáculo: la ausencia de ciudadanía.

Pero este sindicato no fue el único que se les unió. En realidad, fue un numeroso grupo de asociaciones de trabajadores y colectivos de

13 Desde que fundó su colectivo feminista en 1924, Cáceres utilizó en su discurso la idea de la esclava para aludir a la falta de derechos de las peruanas. Inspirada por la obra del inglés John Stuart Mill, en sus artículos, manifiestos y misivas hacía hincapié en la situación de inferioridad en la que se encontraban sus compatriotas: "La esclavitud de la mujer pesa sobre el Perú, impidiéndole surgir" (Cáceres, "Manifiesto" s/p). 
diversa índole los que hicieron más fuerte la protesta ${ }^{14}$. Tal apoyo no hubiera sido posible sin una organización adecuada, que contempló la designación de un comité ejecutivo de la huelga, cuyos integrantes eran Blanca Vigil -telefonista-, Zoila Teresa Noel -presidenta de la Sociedad Cultural de Empleados de Comercio- y Ernesto Cortés -Sindicato de Empleados Bancarios-. Además de la asesoría de Zoila Aurora Cáceres, el abogado Ernesto Barreto Gutiérrez representaría su causa ("La huelga de las telefonistas continua latente" 4).

Contemplaron también a un grupo de "voceras" que entablaron contacto con las redacciones de los periódicos. Ellas eran las que mantenían actualizada la información y agradecían a los redactores la difusión de dicho material. Esto fue destacado, en más de una oportunidad, por el periódico La Crónica, que publicó la fotografía de la comisión que visitó sus instalaciones el 9 de septiembre. Aunque la imagen carece de un rótulo en el que se especifiquen los nombres de las nueve telefonistas, resulta ser un interesante registro visual de un grupo de trabajadoras urbanas, vestidas con trajes y sombreros a la moda, provenientes en su mayoría de la clase media (Figura 3).

14 Entre los adherentes a esta lucha, que fueron sumándose día a día, se encuentran: Asociación de Contadores, Asociación de Telegrafistas, Asociación de Maestros Primarios de Lima, Confederación General de Trabajadores del Partido Laboralista, Empleados de Agua Potable de Lima, Federación de Choferes y Anexos del Perú, Federación de Empleados Bancarios, Federación de Empleados de Hotel y ramos similares, Liga Bondad y Cultura, Parido Laboralista, Radio Telegrafistas, Sindicato de Empleados Apristas, Feminismo Peruano, Centro Feminista $N^{\circ}$ 1, Asociación General de Empleados del Callao, Sindicato Central de Generación Chosica, Yanacota y Santa Rosa, Federación de Estudiantes del Instituto Pedagógico, Sociedad Nacional de Obstetrices, Sociedad Cultural, Sociedad de Empleados de Comercio, Sociedad Cultural Chancay, Federación de Motoristas, conductores y anexos, Confederación de Artesanos Unión Universal y Federación de Panaderos "La Estrella del Perú". 


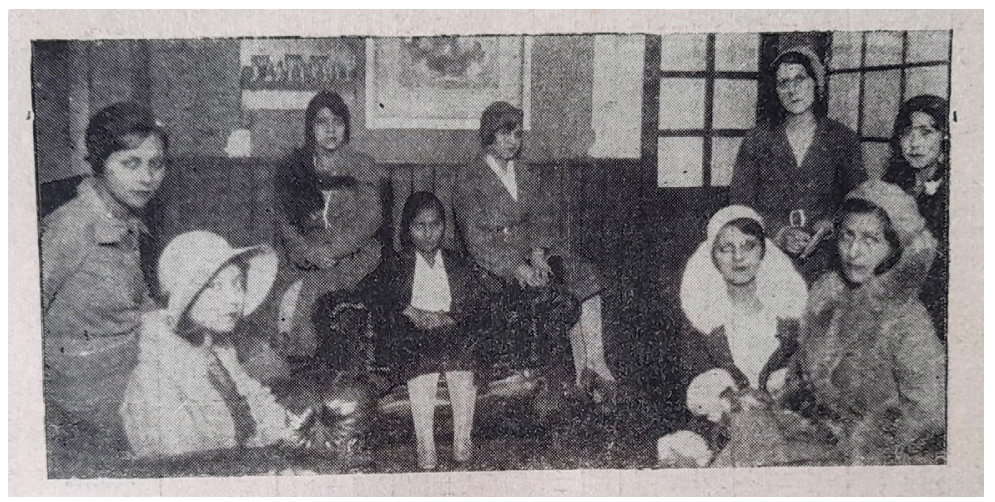

Figura 3. Periódico La Crónica, 10 de septiembre de 1931.

Que el Presidente de la Junta de Gobierno, David Samanez Ocampo, haya llegado a intervenir es una forma de sopesar la resonancia de esta huelga. Esto sucedió el quinto día, cuando una comitiva de telefonistas fue recibida en el Palacio de Gobierno. El grupo de trabajadoras dejó el pliego de reclamos, del cual se destacan cuatro puntos: 1) Reconocimiento del Sindicato de Trabajadores de Telefonistas de Lima, Callao y balnearios, 2) Incremento Salarial (ochenta soles mensuales), 3) Mejora de las condiciones laborales (jornadas de 6 y 5 horas, de acuerdo al trabajo diurno o nocturno; supresión del horario rotativo, implantación de asientos con espaldares, el restablecimiento de gratificaciones, el abono de pago adicional por horas extras, días domingos y feriados) y 4) Compromiso de la reposición de las empleadas despedidas y de la ausencia de represalias a las operarias en huelga ("La huelga de telefonistas continua en el mismo estado" 6).

En tanto, Zoila Aurora envió otras misivas en las que defendía los intereses de las telefonistas. La más contundente fue la publicada el $12 \mathrm{de}$ septiembre en El Comercio, en respuesta a una editorial del mismo diario. En una extensa carta basada en la "política social", expresó su parecer sobre el capitalismo implantado en el Perú y sus consecuencias, que lo habían convertido en el "aniquilador de la vida del trabajador". Ello la hacía pronunciarse a favor del sindicato formado por las telefonistas, del que "surgirán justos reclamos hasta obtener la ley que proteja contra el abuso y la explotación" (Cáceres, "Sobre la huelga" 6). 
Esa abierta postura a favor de los trabajadores le valió a Cáceres ser reconocida como una defensora de los derechos sindicales. Esto lo expresó Alindor Mujica, secretario del Sindicato Central de Generación Chosica, Yanacota y Santa Rosa, en una misiva en la que exaltó su valentía y la destacó como alguien que "conoce muy de cerca la sicología y sus costumbres del obrero nacional” (Mujica, "Carta a Cáceres").

A estas halagadoras palabras, Zoila Aurora no se mantuvo indiferente y en su respuesta agradeció el "voto de aplauso" y renovó su postura a favor de la sindicalización ${ }^{15}$. En lo referido a las telefonistas dijo que, a pesar de "los tropiezos" que encontró en las esferas oficiales, consiguió encausar sus reclamos, los mismos que esperaba llegaran a favorable solución "debido a la intervención que solicité y obtuve del Consejo Municipal” (ibid.).

Esta última línea es un dato de interés dado que Cáceres era amiga del alcalde, José de la Riva Agüero. En efecto, el Concejo Provincial de Lima tuvo una participación decisiva en este conflicto, primero, al organizar una comisión investigadora para determinar las infracciones de la Compañía de Teléfonos, por cuyo resultado fue multada; y segundo, al convocar una conciliación para, finalmente, llegar a determinar un arbitraje. Este resolvió admitir diez puntos del pliego de reclamos, referidos al incremento del sueldo, a la eliminación del horario rotativo y el servicio de meritorias, a la regulación de horas diurnas -descanso de quince minutos durante la jornada-y nocturnas -con refrigerios-, el acondicionar asientos cómodos, no tomar represalias contra las huelguistas y abonar sus honorarios de los días del conflicto. No obstante, el punto más significativo, que fue rechazado, era el concerniente al reconocimiento del sindicato, dado "que el ramo de teléfonos no puede sindicarse sin peligro grave de la seguridad del Estado" ("Definitivamente quedo ayer" 5).

\section{Conclusiones}

Siete años después de la huelga de las telefonistas y de su campaña por el voto de las peruanas ${ }^{16}$, Cáceres publicó la memoria de FPZAC. Allí

15 Se conoce que Cáceres también asesoró al Sindicato de Costureras del Estado, pero de este apoyo no ha quedado mayor registro.

16 La campaña de FPZAC tuvo un resultado ambiguo. Se logró el voto municipal 
escribió sobre su apoyo a la lucha de esas trabajadoras del cable, señaló haber recibido hostigamiento y, sin tapujos, identificó a quienes estuvieron detrás de esas acciones: el ministro de Gobierno, José Francisco Tamayo, y el ministro de Fomento, Ulises Reátegui, "amigo de la Compañía de Teléfonos”. Sobre este desagradable incidente dijo:

El Doctor Tamayo, mediante sus agentes de policía atropelló mi domicilio, en el que se habían reunido las telefonistas al declararse en huelga, por lo que protesté en la Prensa local; al mismo tiempo comprendí, que se corría un riesgo del que quise prevenir a las socias del FPZAC, por lo que continué sola para no comprometerlas, alentando a las valerosas jovencitas que dieron el primer grito de rebeldía femenina lanzando un clamor de justicia (Cáceres, Memorias 9).

Esta actitud intolerante y abusiva evidencia, como en todos los estudios de género, el poder que discrimina y atenta contra las mujeres (Scott 289). En este caso, fue un poder tripartito: el poder represivo de los policías que cumplían órdenes, el poder político, representado en los ministros de Estado, y el poder económico de la Compañía de Teléfonos. También tuvo protagonismo el poder de la prensa, pues la huelga de telefonistas ocupó varias páginas que informaron, día a día, de los avances y retrocesos de las negociaciones. Dichos artículos traslucen opiniones a favor o indiferentes a la lucha de las trabajadoras, como se aprecia en El Comercio y La Crónica, medios que han servido de fuente para este artículo. Mientras el primero trató de mantener una línea imparcial, el segundo no ocultó su simpatía por el reclamo de las telefonistas.

No obstante, estos poderes no amilanaron a las mujeres de esta historia, las huelguistas y Zoila Aurora Cáceres, quienes hicieron uso de la agencia de tres maneras: 1) buscaron y afianzaron la red femenina al unir el reclamo por la ciudadanía con la lucha laboral, 2) establecieron contacto con colectivos de diversa índole para difundir y fortalecer su

de las mujeres, pero nunca se llevó a cabo pues dichas elecciones se establecieron recién en 1963. Es por ello que la primera vez que las peruanas lograron ejercer su derecho al voto fue en las elecciones generales de 1956, luego de expedida la Ley $\mathrm{N}^{\circ} 12391$. Zoila Aurora Cáceres continuó activa en su objetivo de alcanzar la igualdad de derechos de sus compatriotas hasta la década de 1940. 
propuesta y 3) sensibilizaron a hombres y mujeres, al entablar contacto con medios de prensa que reproducían sus objetivos.

De igual manera, la huelga de telefonistas de 1931 abre la posibilidad de un espectro mayor de análisis que conecta el pasado y el presente. Las condiciones de los trabajadores(as) y el abuso de las autoridades que representan a empresas extranjeras, instaladas en países de leyes blandas e indolentes al abuso de sus compatriotas ${ }^{17}$, son, lamentablemente, temas de los que todavía se discute en la actualidad.

Lo anterior no ensombrece las luchas que se entrecruzaron en esos días de 1931, las de Feminismo Peruano Zoila Aurora Cáceres y las de las telefonistas en huelga. Ambas desafiaron el statu quo con tácticas que ponían en evidencia que, aunque las mujeres continuaban bajo un Código Civil del siglo XIX, sus reclamos y necesidades ya eran de mujeres del siglo XX y XXI.

\section{BiBLIOGRAFÍA}

Basadre, Jorge. Historia de la República del Perú (1822-1933). Lima, Empresa Editora El Comercio, 2005.

Belvedresi, Rosa. "Historia de las mujeres y agencia femenina: algunas consideraciones epistemológicas". Epistemología e Historia de la ciencia, vol. 3, N $\mathrm{N}^{\circ}$ 1, 2018, pp. 5-17. Visitado el 10 de junio de 2021. Disponible en: https://revistas.unc.edu.ar/index.php/afjor/article/ view/19865/21594.

Both, Frida. Mujeres y movimiento obrero en Lima 1900-1930. Lima, Biblioteca del Centro Flora Tristán, s/f.

Cáceres, Zoila Aurora. "Programa de Principios de Feminismo Peruano". La Prensa, 2 oct. 1924, p. 9.

17 En el caso de la huelga de las telefonistas, alguien que representó esta postura fue el peruano Fernando Carbajal, gerente de la Compañía Peruana de Teléfonos, quien a lo largo de los días del conflicto mostró ser una persona intolerante y despectiva frente al reclamo de las trabajadoras. 
. "Feminismo Peruano. Contestación a la carta abierta dirigida a la Señorita Zoila Aurora Cáceres”. El Pueblo, 11 dic. 1930, p. 11. . "Manifiesto de Feminismo Peruano ZAC". Lima, correspondencia de Zoila Aurora Cáceres en la Biblioteca Nacional del Perú, 1931.

. "Manifiesto de Feminismo Peruano ZAC". Los Andes, 11 mayo 1931, pp. 2-3.

. "Sobre la huelga de telefonistas". El Comercio, 12 sep. 1931, p.12.

. "Carta a Alindor Mujica", 19 sep. 1931, correspondencia de Zoila Aurora Cáceres en la Biblioteca Nacional del Perú.

. Memorias de la Presidenta del Feminismo Peruano ZAC. Lima, Imprenta La Confianza, 1938.

Carlessi, Carolina. Mujeres en el origen del movimiento sindical. Lima, Editorial Lilith y Tarea, 1984.

"Continua sin solución la huelga de telefonistas de Lima”. La Crónica, 28 ago. 1931, p. 11.

Contreras, Carlos y Marcos Cueto. Historia del Perú Contemporáneo. Lima, Instituto de Estudios Peruanos, 2013.

Chávez Aliaga, Nazario. "La movilización política de la mujer". La Crónica, 13 de jul. 1931, p. 11.

De la Cruz, Luis. "Aquellas telefonistas de armas tomar." el diario.es, feb. 7, 2015. Web. Visitado el 7 de diciembre de 2020. Disponible en: https://www.eldiario.es/madrid/somos/malasana/aquellas-telefonistas-de-armas-tomar_1_6412406.html

Del Prado, Josefina. "Consecuencias de la Primera Guerra Mundial en el Perú". El Perú y la primera Guerra Mundial, Fabián Novak y Jorge Ortiz (eds.), Lima, Fondo Editorial de la PUCP, 2014, pp. 195-218.

"Definitivamente quedo ayer solucionada la huelga de telefonistas." $\mathrm{La}$ Crónica, 29 sep. 1931, p. 5.

"El estado de la huelga de telefonistas". La Crónica, 9 sep. 1931, p. 12.

"El servicio telefónico se inició en Lima con tres abonados y cuenta hoy con más de 9000". El Pueblo, 4 dic. 1930, p. 10.

Felacio, Laura. "La huelga de las telefonistas. Condiciones, problemas y manifestaciones de las mujeres obreras a comienzos del siglo XX en 
Bogotá". Ciudad Paz-ando, vol. 5, № 1, 2012, pp. 31-48. Visitado el 15 de junio de 2021. Disponible en: https://revistas.udistrital.edu. co/index.php/cpaz/article/view/7273/8963

Huamani, Edilberto. La telefonía en el Perú 1888-2008. Lima, Fondo Editorial Eduni, 2020.

"La huelga de telefonistas". El Comercio, 27 ago. 1931, p. 12.

"La huelga de telefonistas". La Crónica, 6 sep. 1931, p. 17.

"La huelga de telefonistas continúa en el mismo estado". La Crónica, 1 sep. 1931, p. 6.

"La huelga de las telefonistas continua latente". La Crónica, 29 ago. 1931, p. 4.

Martínez de La ToRre, Ricardo. Apuntes para la interpretación marxista de la historia social del Perú. Lima, Compañía Impresora Peruana S.A., 1949.

Mujica, Alindor. Carta a Zoila Aurora Cáceres, 10 abril 1931, correspondencia de Zoila Aurora Cáceres en la Biblioteca Nacional del Perú.

Pachas, Sofía. Zoila Aurora Cáceres y la ciudadanía femenina. La correspondencia de Feminismo Peruano. Lima, Fondo Editorial de la UNMSM, Jurado Nacional de Elecciones y Centro de la Mujer Flora Tristán, 2019.

Padilla, Cristian. Cultura, espectáculo e institucionalidad politica en el Perú: El proceso electoral general de 1931. Tesis para optar el título de licenciado en Historia, Universidad Nacional Mayor de San Marcos, 2019.

Pellegrini, Micaela. "Huelgas, maestras y rumores. Una escena de mujeres en lucha a partir de un estudio de caso (Firmat, Argentina, 1921)". La Ventana, vol. 6, N 49, 2019, pp. 283-316. Visitado el 14 de junio de 2020. Disponible en: http://www.scielo.org.mx/pdf/ laven/v6n49/1405-9436-laven-6-49-283.pdf

"Permanece en el mismo estado la huelga de las telefonistas." La Crónica, 30 ago. 1931, p. 9.

Perrot, Michelle. "Salir". Historia de las mujeres. Tomo 8. El siglo XIX. Cuerpo, trabajo y modernidad. Georges Duby y Michelle Perrot (eds.), Madrid, Taurus Ediciones, 1993, pp. 155-189. 
Ravines, Eudocio. “Carta a Zoila Aurora Cáceres”, 17 sep. 1931, correspondencia de Zoila Aurora Cáceres en Biblioteca Nacional del Perú.

Rojas, Joel. "Estudio preliminar. Miguelina Acosta Cárdenas: pensamiento y acción”. Miguelina Acosta Cárdenas. Escritos selectos. Joel Rojas (ed.), Lima, Heraldos Editores, 2020, pp. 9-16.

Sсотt, JoAn. "El género: una categoría útil para el análisis histórico". El género: la construcción cultural de la diferencia sexual, Marta Lamas (comp.), México, PUEG, 1996, pp. 265-302.

"Se declaran en huelga las operadoras de teléfonos." La Crónica, 27 ago. 1931, p. 12.

Vidaurre, Ernesto. "La huelga de las telefonistas". La Crónica, 27 ago. 1931, p. 12.

. "Las causas del mal servicio telefónico y su remedio". $\mathrm{La}$ Crónica, 31 ago. 1931, p. 6.

Villavicencio, Maritza. Breve historia de las vertientes del movimiento de mujeres en el Perú. Lima, Centro de la Mujer Flora Tristán, 1990.

Yael, Dina y Silvana Darré. "El triunfo de las señoritas telefonistas. El primer sindicato de mujeres del Uruguay y el impacto de la huelga de 1922". Revista Zona Franca N² 28, 2021, pp. 270-302. Visitado el 14 de junio de 2021. Disponible en: https://zonafranca.unr.edu. ar/index.php/ZonaFranca/article/view/166/177

Zegarra, Margarita. María Jesús Alvarado. La construcción de una intelectual feminista en Lima (1878-1915). Lima, Fondo Editorial del Congreso del Perú, 2016.

Recepción: 14-01-20

Aceptación: 21-05-20 